\title{
A SuRvey OF MODELS FOR COMPUTER NETWORKS MANAGEMENT
}

\author{
Dimitris Kontoudis ${ }^{1}$ and Panayotis Fouliras ${ }^{1}$ \\ ${ }^{1}$ Department of Applied Informatics, University of Macedonia, Thessaloniki, Greece
}

\begin{abstract}
The virtualization concept along with its underlying technologies has been warmly adopted in many fields of computer science. In this direction, network virtualization research has presented considerable results. In a parallel development, the convergence of two distinct worlds, communications and computing, has increased the use of computing server resources (virtual machines and hypervisors acting as active network elements) in network implementations. As a result, the level of detail and complexity in such architectures has increased and new challenges need to be taken into account for effective network management. Information and data models facilitate infrastructure representation and management and have been used extensively in that direction. In this paper we survey available modelling approaches and discuss how these can be used in the virtual machine (host) based computer network landscape; we present a qualitative analysis of the current state-of-the-art and offer a set of recommendations on adopting any particular method.
\end{abstract}

\section{KEYWORDS}

Network Management, Modelling, System \& Network Virtualization, Hypervisor

\section{INTRODUCTION}

The virtualization concept has been of interest to the academic community and IT business sector for more than forty years, providing a different approach to the realization, administration and provision of physical resources. A new paradigm is introduced by which the end user is not aware of the details of the underlying physical infrastructure. This paradigm has been warmly adopted in many fields of computing, including computer networks. The Internet - being the prime example of a large scale and complex network instantiation [1] - has been the driving force behind the adoption of network virtualization as one of the key technologies in the field [2-4]. Other trends in networking and computing increase the complexity of network environments, mainly, due to three reasons:

1. the characteristics of modern network implementations (size, operational and other).

2. the inevitable convergence of two distinct worlds: communications and computing. The use of computing servers acting as active network elements (e.g. as routers) is becoming increasingly popular [7-10]. The core networking support in these network environments is based on the IEEE 802.1Q VLAN implementation, where virtual network segments are established on top of physical switches - the latter being provided by the server's hardware features (a thin software layer - the hypervisor - works as a virtual Ethernet switch and supports queues for each VLAN in the system's memory). The network's last-hop switch has, consequently, been shifted from a dedicated active network element to become a characteristic of the hypervisor or of the physical server's hardware [11-13].

3. the application of new technologies that lead to demand for novel services (wireless/mobile

DOI : $10.5121 /$ ijcnc.2014.6313 
networking, cloud computing, networking as a service etc.) [14-19]. The complexity in several [21,22] virtual network implementations will obviously be increased, resulting in impediments in the assurance of quality of service, on an end-to-end basis, unless some standardization approach is applied. Cisco Systems projects that thirty seven billion intelligent devices (some of peculiar nature, e.g. smart fabrics and pills) will connect to the Internet by 2020 - dramatically increasing the traffic load and operational complexity of the "The Internet of Everything" (see Cisco Blogs, http://blogs.cisco.com/ioe/beyond-things-theinternet-of-everything-takes-connections-to-the-power-of-four).

Managing network environments presents an increasing need for the infrastructure detailed characteristics to be represented in a formal, standardized and structured manner, regardless of the representation stakeholders (researchers, providers, end-users). This is facilitated by the use of suitable information and data models that allow for better description of the involved infrastructure components and for the organization of their characteristics and interrelations. Several proposals for such models have been introduced and some have found actual application outside of the research domain. Our goal is to provide researchers with a review of the current state-of-the-art, as assessed from a host-based network resources provisioning angle, along with a comparative discussion that will help in better choosing a proposed approach. We hope that the work presented in this paper will ease the task of conducting relevant research. It is not our intention to suggest any particular proposal, rather than, to introduce and discuss relevant considerations on the topic. Our research differs from surveys on network virtualization $[5,6]$ in that it focuses on the modeling aspect employed in the virtual network architectures, accounting for advances in the host (system) virtualization domain.

Paper Organization: section 2 introduces a generalized overview of the surveyed proposals along with a description of assessment criteria used in this paper. Sections 3-6 provide an introduction on each proposal. Detailed discussion and comparisons are given in section 7. We conclude in section 8 summarizing the findings of our work.

\section{MODELLING PROPOSALS}

\subsection{Overview}

Many parties, industrial, commercial and academic alike, are actively involved in network virtualization research, occasionally in joint ventures. Research spans a wide variety of topics, ranging from very specific technical issues (interfacing, signaling and bootstrapping, resource and topology discovery, resource allocation, admission control, virtual nodes and virtual links, naming and addressing) to broader interest areas such as mobility management, monitoring, configuration and failure handling, security and privacy, interoperability issues. A concise survey, in a holistic and detailed manner, of network virtualization research is provided in [5, 6]. Active [23], programmable [24] and overlay [25,26] networks benefit, as well, from advances in system and network virtualization. Network virtualization architectures are discussed in [27-32].

The state-of-the-art information models available to the systems and computer network domains are the Common Information Model (CIM) [33] (proposed by the Distributed Management Task Force - DMTF [34]), the Shared Information/Data (SID) model [35] (proposed by the TeleManagement Forum - TMForum) and the Directory Enabled Network next generation (DENng) [36] (proposed by the Autonomic Communications Forum [57]). Each of these models, as discussed in later sections, focuses on network aspects from a different perspective. CIM applies a holistic approach conceptualizing computing systems and networks in general, whereas SID and DEN-ng spawn from the telecommunication industry and better represent business (as well as technical details), in the telco context. CIM has been used as the basis for the creation of other 
information models that focus on specific application areas, such as hypervisors and virtual machines [38], and virtual network environment provisioning [39]. An overview of the three main information models can be found in [40] and their use in a network virtualization context is discussed in [41] and [42].

Two other areas of related research can be, collectively, identified as presenting modeling elements. The first area refers to the network description languages (NDLs) [43], some of which do contain small information models and other modeling approaches. These languages have been designed with the goal of imprinting network characteristics in a structured and hierarchical manner. Code developed in any of these languages can be used in a diverse array of applications (e.g. as input to special purpose software). NDLs are, therefore, used as modeling tools for the design and application of abstractions on the physical and logical representation layers of the networking infrastructure. The second area refers to work based on the Management Information Base (MIB) concept [44] - databases storing management information about devices and applications. These databases are populated and used by management applications, using specialized protocols such as the Simple Network Management Protocol (SNMP). MIBs result in data models of managed entities and this concept can be applied to abstract physical and logical device state and configuration as well as application specific information.

Testbed network implementations, pursued in academic and/or private sector (mainly EUfunded) projects, introduce some level of formalization across different layers of their architecture (indicative projects: GENI, Emulab, Planetlab, VINI, Federica, Nitos, Etomic, PanLab, Wisebed, Geysers, Novi and SAIL). Information models can be found in the Novi and Geysers projects with the Novi [41] and LICL [45] proposals respectively. Finally, special mention should be given to Software Defined Networking [46], a new paradigm in network architecture and management - a promising approach [47], with its own data model, in simplifying change facilitation in the network control logic [48]. SDN enjoys broad industry support given the flexibility it offers in data center fabric management.

\subsection{Assessment criteria}

Qualitative analysis of examined work presents difficulties due to the fact that there is no objective and commonly accepted standard by which comparisons can be made. Our assessment proposal uses a number of criteria that, intuitively come into mind and are in use in the field, attempting to distinguish items found in literature based on three main areas: a) the overall positioning of the proposed work, b) the modeling aspects, and c) the system virtualization perspective. In that direction we have defined thirteen distinct criteria, presented below.

\subsubsection{Overall positioning}

Based on a logical categorization we attempt to form a high level grouping, examining the scope and maturity of each proposal - it is, thus, possible to obtain a generalized assessment:

a) group (main/MIB/NDL/other): overall categorization of each proposal. This can be main information models-based, MIB-based, NDL-based or other

b) resources $(T / O / B)$ : items that can be modeled/abstracted/standardized by the proposed approach. These can be of technical, operational or business nature

c) maturity (high/low): assesses the current status of the proposals with regard to actual applications as well as industry and community adoption 


\subsubsection{Modelling aspects}

Modern virtual networks, as discussed, can be very complicated in their design, incorporating a variety of physical and virtual resources (servers, hypervisors, routers, nodes, protocols, etc.) as well as related services (routing, monitoring, etc.) and member elements (providers, users, SLAs, etc.) The diverse variety of information does not always fit into a particular type of model. Each proposed model type can serve a particular purpose, though overlapping functionality is apparent across different model types $[42,49,50]$. Furthermore, a certain number of semantics will enable the model to be in a position to span different contexts and allow for handling varying infrastructure items and operational scenarios $[41,42]$ :

d) model type (I/D): as per the criterion name (informational, data)

e) representation (various): indicates the representation method used

f) views (no.of): support for different perspectives on the abstracted objects

g) policy (yes/no): support for establishing conditions and actions

h) context (yes/no): support for performing actions based on application-specific information

i) capability (yes/no): support for handling and processing different data types and structures

j) state (yes/no): support for capturing different states of the managed environment and for enabling triggering of specific actions based on the state information

\subsubsection{System virtualization perspective}

The use of hypervisors and virtual machines complicates even further the handling of several aspects of the infrastructure [51,52]. Resources that are provisioned via the system virtualization layer need to be addressed in a system-level environment and the specific characteristics need to be incorporated in the model's semantics. Thus, the model needs to include design and logic to account for hypervisors and virtual machines:

k) virtualization (yes/no): support for system (host) based virtualization (virtual machines, virtual CPU/memory etc.)

1) hypervisor (yes/no/indirectly): as per the criterion name - if the proposal explicitly defines semantics for hypervisors

m) hypervisor agnostic (yes or N/A): whether the model can abstract any hypervisor (i.e. not limited to a specific product)

From a presentation perspective, a direct grouping is possible as four high level categories can be used to span all efforts in the field: i) the main information models, with CIM and CIM-based proposals, SID and DEN-ng, ii) MIB-based work, iii) Network Description Languages, and iv) un-generalized approaches (i.e. not falling under a collective category). This presentation path is adopted in the rest of the paper.

\section{THE MAIN INFORMATION MODELS}

\subsection{CIM and CIM-based approaches (3 items)}

The Common Information Model (CIM) [33] proposed by the Distributed Management Task Force (DMTF) is a conceptual, object-oriented, information model for describing the management entities in computing environments. The model is not bound to any particular implementation and, thus, enables the platform-independent and technology-neutral exchange of management information, providing a consistent definition and structure of data. CIM consists of a specification and a schema. The specification describes the model's integration aspects, core 
architecture and basic interrelationships. The schema consists of an extensive set of modeled entities covering areas such as systems, networks, devices, virtualization, applications, metrics and other. All components of CIM (the specification, the schema and the schema extensions) are expressed and maintained in the Open Management Group's Unified Modeling Language (UML) [53]. By using the CIM concept it is possible to manage systems through the use of management applications and the interchange of management information between them through the Common Information Model Object Manager (CIMOM), which is an object management engine that exists between the managed system and the management application. The primary example of explicit network-related modeling can be found in the CIM Network model [54]. This characterizes a network as a type of administrative domain, which may contain other networks or sub-networks in a recursive relationship. The model covers both generic aspects required to represent connectivity between systems and relationships to the underlying physical components, as well as network technology and protocol specifics. The CIM Network schema can be extended to model specific network instantiations and architectures. Virtual system configurations can be modeled in several contexts using the CIM System Virtualization and Virtual System profiles [55,56] (for host systems and discovery of hosted virtual systems used as active network nodes and for in depth representation of a virtual system and its components, respectively). Detailed modeling includes hardware and logical device resources (CPU, memory, networking adapters, etc.) and some methods against those entities (basic control operations).

In [39] the authors propose the VNE-CIM information model for the formal specification of Virtual Network Environments (VNEs). These are described as a collective of interconnected virtual devices in a certain topology, regardless of the underlying system virtualization platform (Xen, VMware, KVM, etc.) The model includes mapping of VNE creation, provision and administration procedures as well as several different system virtualization platforms (used as hosts - virtual nodes - in NVEs). The model has been applied, as an initial proof of concept, on a system setup based on XEN and VMware virtualization platforms, together with the use of opensource libraries for DMTF CIM instrumentation. The current version of the VNE-CIM approach does not model virtual link characteristics (bandwidth, delay, packet loss, etc.) or cross-node VNE deployment based on each node's available resources.

The work in [38] introduces the KF information model, which can be applied for representing any physical or logical element that can be instrumented by CIM and used in a virtual network implementation (networking, computing system or other resource). KF, still at an early stage, is promising in the sense that it has been designed with the system virtualization aspect as the primary focus and, thus, has explicit abstractions for hypervisors and their built-in IEEE virtual switches. The model is demonstrated for the creation of a mechanism for the management of the overall computing system's resource utilization, as it is provisioned for the virtual network instantiation. Furthermore, extensibility of the model is illustrated with the inclusion of Statistical Process Control methods for guaranteed system performance delivery.

\subsection{Shared Information/Data (SID) model}

The Shared Information/Data (SID) model [35] has been proposed by the TeleManagement Forum (TMForum), an international consortium of communication services providers, network operators and relevant suppliers of equipment to the telecommunications domain (hardware and software). The model is, nowadays, part of TMForum's NGOSS Frameworx suite [58]. While SID spawns from the networking world, it mainly covers business entities and processes along with the information flow between them, targeting the enablement of operational support systems and the flawless integration of systems and telecom operation processes. SID essentially is a composition of various industry models and presents a common information language for describing management data pertaining to the telecommunications industry. 
SID is an information and a data model along with a common business and system vocabulary allowing for the coverage of telecommunication business context by means of a high level classification of entities (realized in a framework consisting of different concept areas know as Domains) together with their attributes and interrelations. SID defines domains at a progressing level of detail, each domain designed as being self-contained with links to other domains. Class breakdown (the model includes approximately 1000 classes) ranges from general, abstract, concepts ("Product", "Service", "Customer", "Resource", "Partner" etc.) to very specific ones. The increasing level of detail is represented via the Aggregate Business Entities (ABEs) and their tied information and operations. The implementation of the actual functionality can make use of standardized input and output (e.g. XML files) that result from the use of the model. In such way, the data fed to different applications is very well formalized. Cross-area functionality benefits from this common description of concepts and assets. However, the large number of subclasses used under the root class, as in the case of DMTF CIM [33], can lead to confusion and possibly complicate the use of the model. Elements and the relationships between them are expressed in UML, the widely adopted standard for building information models. Furthermore, model definitions do include XML schema definition representations (XSD), thus providing the ability for reusable data models. The SID model is data representation agnostic and does not follow or endorse any particular approach (e.g. a database system or class diagrams).

\subsection{DEN-ng}

The Autonomic Communications Forum DEN-ng (Directory Enabled Network next generation) information model [36] has its roots in the network management (specifically in policy based network management - PBNM [50]) and in the autonomic networking [59] areas. The focus of PBNM has been to construct information models for the representation of policy and its specifics, whereas autonomic networking concentrates on creating networks that are selfadjusting so as to adapt to changing needs, based on policy and context. Policy can be conceptualized as a control entity with specific attributes related to the managed environment [60]. The DEN-ng model, in its conceptualization, development and application, has significant ties to the OSS/BSS environments in the telecommunication industry and to other related efforts (for instance to the TMForum's NGOSS Frameworx suite [58], where DEN-ng views are identical to the NGOSS views. Furthermore, TMF SID derives in part from an older version of DEN-ng.

DEN-ng is an object oriented information model which provides a common way for the representation of management information (devices, services, users etc.) allowing for policy and the context within it is applied to be taken into account. Description of managed entities is done from different perspectives (views): business, system, implementation and deployment. The model uses software patterns and roles for modeling managed entities. Information in the model is organized via the use of a single root class with three subclasses (Entity, Value and MetaData). These form the top-level hierarchies via which general semantics are gradually fine-tuned and made specific by subclassing and addition of more detailed data, as the need arises. Special classes and associations (Context, PolicyConcept) allow for handling policy $[61,62]$ and context [63] in the desired detail. It is important to note that DEN-ng models characteristics of any handled entity, not the entity itself as a self-contained item (the latter approach is used in CIM model). This, primarily, allows for reusability of the created components. The DEN-ng model is extensible and, thus, can theoretically cover all aspects of a network and its operational environment, including network virtualization features and technologies. A specific application in a virtual network context was pursued under the Autonomic Internet Project framework (AUTOI) $[64,65]$. In this project, management overlays were defined for controlling virtualized resources and related services. 


\section{MANAGEMENT INFORMATION BASES (MIBS)}

A Management Information Base (MIB) is a logical information store consolidating entity details, organized in a hierarchical (tree-structured) manner. While mostly found in the network management and monitoring context $[66,67]$ MIBs are also used in other areas such as computer systems and high-availability cluster management. Accessing a MIB involves using a specialized protocol (Fig. 1), most often the Simple Network Management Protocol (SNMP). The actual properties of the managed objects are populated into the MIB information store by means of specialized software (the MIB module) that implements, most often, then SNMP protocol. Once the MIB database is populated the management applications can access it and retrieve the device data.

In network management MIBs provide a tool towards abstracting the operational information, statistics and status of the physical or virtual devices layer from the part of the infrastructure that needs to access it. The resources that can be modeled include physical [68] and logical [69] devices as well as the software that runs on them. Functional details and interrelations, such as user roles, cannot be handled. The outcome is a management information model which describes technical and operational aspects of the network infrastructure. Numerous network virtualization approaches make use of MIBs, including virtual networks and virtual routers, overlays, Multiprotocol Label Switching (MPLS) VPNs, Multi-Virtual Route Forwarding (VRF) and Policy Based Routing (PBR) [70-73].

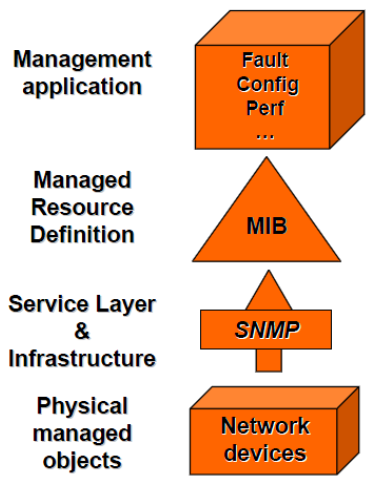

Fig.1. The MIB management concept

If one needs to apply MIBs for managing a virtual network in a system virtualization context, the most prominent approach is the combination of different MIBs. To better illustrate this limitation consider IETF's Entity-MIB [69]. This is a well suited common candidate for hardware infrastructure targeted management as it can handle information between logical entities and physical entities on one network element as well as information about the hierarchy among physical entities. However, the detailed architecture of the physical infrastructure cannot be thoroughly represented. To overcome this limitation, another MIB, the Physical Topology MIB [68], can be related to the Entity-MIB and introduced into the model so as to provide the missing semantic coverage. This approach is necessary in order for a MIB-based model's scope to be broadened.

\section{NETWORK DESCRIPTION LANGUAGES (NDLS) (5 ITEMS)}

Description languages, in general, are tools (formal languages) that allow for the high level description of a system and its properties. These languages aid in the planning, systemic analysis 
International Journal of Computer Networks \& Communications (IJCNC) Vol.6, No.3, May 2014

and resource specification of the described system. The output of a description language is, most often, XML or XML-based [74] code that can be used as input in certain parsing software. Several such network description languages (NDLs) exist in the computer networks field primarily focusing on physical rather than virtual networks [43]. Virtual networks, though, present special attributes (as it has been discussed in the previous sections) that are not, currently, within the scope of available NDLs. The latter, nevertheless, can be of use in modeling as any standardization approach presupposes the formal and structured element description. It is in this direction that NDLs can be employed using their representational abstractions, like ontologies and logical schemas that support conceptual infrastructure imprinting.

The Network Description Language (NDL) [75] is the most prominent among the relevant proposals. It is being maintained by the System and Network Engineer Research Group (SNE) of the University of Amsterdam in the Netherlands. NDL is based on the RDF language - a metadata data model, part of W3C consortium's specification for the theoretical description and modeling of Internet resources [76]. NDL, essentially, is an information model which includes ontologies and schemas (represented in UML) allowing for simple, yet, comprehensive network descriptions. Network information is categorized in network topologies, technology layers, device configurations, capabilities and topology aggregations. The language defines several schemas for the description of different aspects of the network infrastructure, as per the aforementioned categories (topology, layer, capability schemas). A domain schema describes administrative network domains and abstractions for contained devices, and the physical network infrastructure is described by the relevant physical schema. NDL has found particular application in the definition of network topologies [77,78] and the relevant processing of those definitions for creating network maps [79] or discovering specific resources (such as paths) [80].

The Network Markup Language (NML) has been proposed in [81] by the Open Grid Forum's (OGF) NML Working Group [82,83] and is a description language whose conceptual design consist of flexible and extensible schemas, allowing for the inclusion of network attributes and the construction of new ontologies, as new elements and demands become available. NML focuses on connection oriented topologies of physical and virtualized networks, especially, on service discovery and provisioning issues in such architectures. The language does not describe aspects such as policy, scheduling and reservation (these types of aspects are, currently, outside NML's design scope). The UML language schema describes layer independent network topologies and the properties common to different technologies employed in the infrastructure [84]. A network description in NML is expressed in XML and RDF. Examples uses of the language include optical path finding and resource/topology inventory description.

The Virtual Grid Description Language (vgDL) [85] was developed at Rice University under the VGrADS project and pertains to the Grid Computing area. vgDL provides a framework for the abstractive description of resources, especially related to Grid applications. Resource specification in $\mathrm{vgDL}$ consists of a core resource description along with a ranking function describing the conditions under which the resource is needed. Specific associations indicate resource interdependencies. vgDL example uses include describing the attributes and characteristics of workflows in Grid environments [86,87].

The Virtual Resources and Interconnection Networks Description Language (VXDL) [8890] originates from the Grid Computing area (like vgDL). Grid implementations present special operational characteristics - in a Grid environment it is imperative that resources involved can be dynamically re-allocated, without disturbing any running applications. These resources can be of many types: virtual machines acting as nodes, interconnections, network bandwidth etc. The language allows for the description of the virtual infrastructure resources, the network topology and their specific attributes. It is worth noting that elements of VXDL and NDL were used as base components for the LICL information model [45] of the Geysers project. 
The proposal in [91] is the Infrastructure and Network Description Language (INDL), a recent effort aiming at describing computing infrastructures in a technology independent way. The language provides semantic descriptions for physical resources along with their virtualization mechanisms and the underlying networking infrastructure and can be extended to include other aspects, such as infrastructure federations, resource behavioral aspects etc. INDL is related to research results and activities from NML-WG [83], the GEYSERS [124] and NOVI [41] projects. One of the main approaches used for INDL's conceptual schema was the Geysers Information Modeling Framework [45].

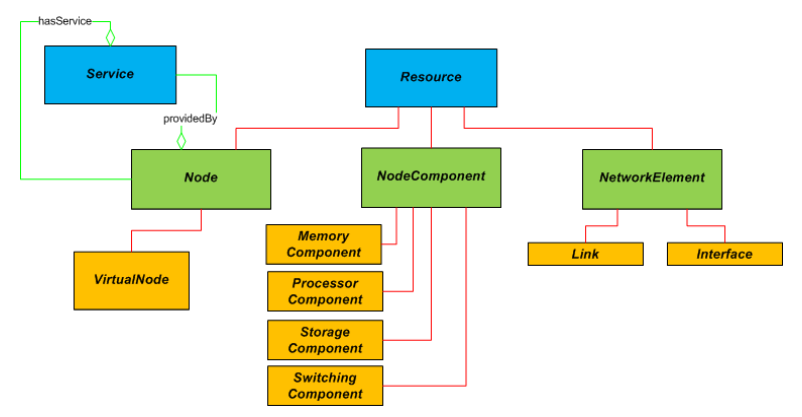

Fig.2. The INDL class hierarchy

INDL is based on an ontology which defines a hierarchy of abstract classes, their associations and properties (Fig. 2). Two main classes (Resource and Service) are used for describing the core components and characteristics of a network setup. Three further sub-classes (Node, NodeComponent, NetworkElement) allow for modeling physical/virtual nodes, their characteristics (cpu, memory etc.) and network connectivity respectively. Virtualization specifics are modeled via Node's special subclass VirtualNode. The concept of a service is abstracted at the participating node level which is considered as either owner or provider of a service, depending on the view applied on the class.

\section{OTHER PROPOSALS (6 ITEMS)}

In [41] authors propose NOVI (Networking innovations Over Virtualized Infrastructures), an information and data model targeted at describing virtual resources in federated heterogeneous Future Internet platforms. The model facilitates management of such infrastructures by enabling data handling, resources control, provisioning and monitoring. Rich in modeling features NOVI includes semantics and support for resource state handling, context-awareness and managements policies, and instantiated in a virtualized infrastructure context.

The LICL (Logical Infrastructure Composition Layer) information model, developed under the Geysers project, is proposed in [45] and focuses on physical resource to virtual infrastructure provisioning and management. The infrastructure layer is described via a series of physical to logical resource abstractions incorporating host-based virtualization as end-point network elements. The core aim of the LICL model is to allow decoupling of the infrastructure (specifically, resource management) from the service provisioning.

The Node Architecture is proposed in [92] (under the 4WARD project [93]) which provides a framework for network virtualization, incorporating design for commercial environments and business scenarios. An RDF-based data model has been developed for the construction of the VNet virtual network, along with its characteristics and available resources. Several networking 
physical resources can be virtualized in the context of the VNet, such as routers, wired and wireless links. Provisioning, management and control of the VNet has been demonstrated in different scenarios. Finally, a flexible XML-based scheme has been provided for network description. 4WARD, and other Future Internet research projects, are based on the InformationCentric Networking (ICN) concept [94]. The EU-funded SAIL project (Scalable and Adaptive Internet soLutions) [95] adapts to the ICN concept and builds upon the research artifacts, primarily, of 4WARD in order to design architectures, technologies and techniques for adapting current network infrastructures to Future Internet concepts.

Authors of [96] propose the Virtual Network Specification Schema (VN-SLA), an XML-based schema for abstracting a network architecture's business resources and their interrelations, including virtual network provisioning scenarios along with the parameters inherent in their design. A top-level VN-SLA class is defined which contains the abstraction and details of a Service Level Agreement (SLA) in a virtual network provision scenario. Focus is on resources provisioned by infrastructure providers (InP) and virtual network providers (VNP), supplying a basis for describing virtual resources and virtualization services at the level required for automated virtual network provisioning.

The VNMI proposal presented in [97] and submitted as a working document to the Internet Engineering Task Force introduces an information model for the management of virtual switches. The model describes the physical layer (connections between physical switches) and the virtual layer (connections between virtual switches) in the networking infrastructure. These layers represent the association of the virtual switch with the corresponding physical switch. The approach is focused on device management and, from this perspective, can model physical and virtual resource and hierarchy (mapping) information between members, along with the relevant technical characteristics. Implementation of the model will result in a MIB-based approach and, consequently, usage of other MIB elements will be necessary in order to extend the model's scope.

Software-Defined Networking (SDN) [46] presents a new, industry popular, paradigm in network architecture by which the network control and data planes are decoupled [98]. The network's intelligence and state are separated from the core components (routers and switches) and are consolidated on control entities (computing servers) [99]. This decoupling (Fig. 3) provides the benefit of having different distribution models and actual implementations of the two planes [100, 101]. SDN has evolved, as one may have expected, in close progress with developments in server virtualization and recent trends in computer networks (ossification of the Internet, cloud computing, extensive user mobility, etc.) 


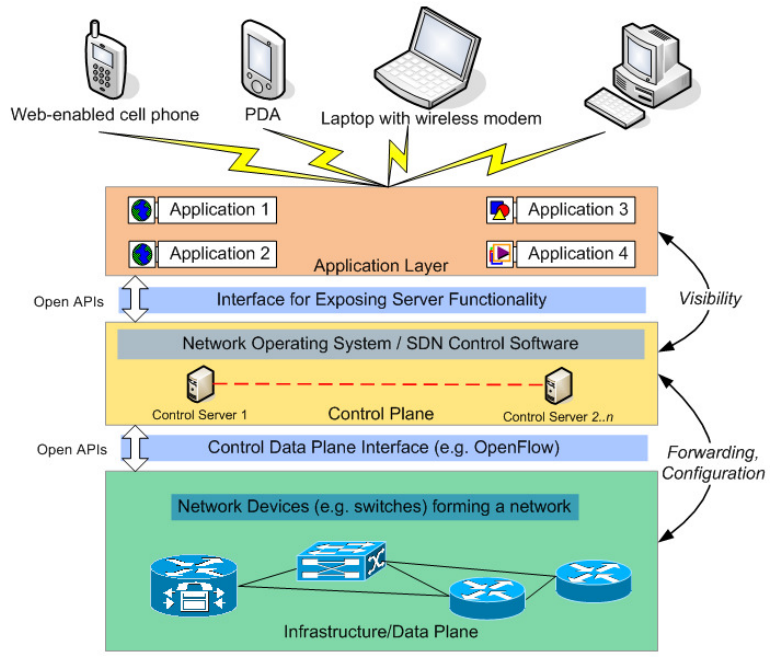

Fig.3. Logical view of the SDN architecture

Configuration and management protocols in the SDN paradigm include OpenFlow [102] and OFconfig. The latter is based on NETCONF [103], an XML-based protocol that provides mechanisms to install, manipulate, and delete network device configuration. The underlying data model is based on YANG [104] and describes the resources that can be configured along with the relations between different resource configurations. SDN being somewhat close to the systems hypervisor concept, in that the latter is ran on actual hardware to abstract it from the operating system, has been referred to as the network hypervisor [105,106].

\section{DISCUSSION}

A model is a developed representation of a real world system. Different types of models exist that can be used for representing structured and unstructured information, relationships and elements in a given environment $[49,107]$. Choosing a proposal for application in a new project involves determining, beforehand, the target use of the model to be created in conjunction with the modeling capabilities offered. Three main factors affect the selection: a) the kind of resources that need to be modeled, b) the requirement for actual implementation in real applications (i.e. the need for instrumentation) and, c) the capabilities of the desired model for describing the infrastructure in question. Virtual network environments can be multidimensional entities with respect to the nature, role and function of their elements. Every proposal surveyed in this paper presents different advantages and disadvantages. From a pure modeling perspective it is clear that the three main information models (CIM [33], SID [35], DEN-ng [36]) are superior in modeling concepts and features than that found in other proposals. These models, though, are complex; they require greater learning effort as well as increased development and maintenance costs, still enjoying broad industry support. Given the diverse nature of infrastructure resources and the variety of available modeling approaches $[108,109]$ it remains to be determined which approach can be best applied for abstracting this large collective of information, relations, and entities. Ultimately, choosing a model will depend on a number of factors, both modeling-related and other (like technical, cost etc.) A summary of the assessment of the proposals is presented in Table 1. 
International Journal of Computer Networks \& Communications (IJCNC) Vol.6, No.3, May 2014

Table 1. Summary of surveyed proposals

\begin{tabular}{|c|c|c|c|c|c|c|c|c|c|c|c|c|c|}
\hline & $\begin{array}{l}\text { CIM } \\
{[33]}\end{array}$ & \begin{tabular}{|c} 
VNE- \\
CIM \\
[39]
\end{tabular} & $\begin{array}{c}\mathbf{K F} \\
{[38]}\end{array}$ & $\begin{array}{l}\text { SID } \\
{[35]}\end{array}$ & $\begin{array}{c}\text { DEN-ng } \\
{[36]}\end{array}$ & $\begin{array}{l}\text { MIB } \\
{[44]}\end{array}$ & $\begin{array}{l}\text { NDL } \\
{[43]}\end{array}$ & $\begin{array}{c}\text { NOVI } \\
{[41]}\end{array}$ & $\begin{array}{c}\text { LICL } \\
{[45]}\end{array}$ & \begin{tabular}{|c} 
NODE \\
A. \\
{$[92]$}
\end{tabular} & $\begin{array}{l}\text { VN- } \\
\text { SLA } \\
{[96]}\end{array}$ & \begin{tabular}{|c|} 
VNM \\
I \\
{$[97]$} \\
\end{tabular} & $\begin{array}{l}\text { SDN } \\
{[46]}\end{array}$ \\
\hline & \multicolumn{13}{|c|}{ Overall Positioning } \\
\hline group & main & main & main & main & main & MIB & NDL & other & other & other & other & other & other \\
\hline resources $^{\mathrm{a}}$ & TO & $\mathrm{T}$ & TO & TBO & TB & $\mathrm{T}$ & $\mathrm{T}$ & TO & TO & TO & $\mathrm{B}$ & TO & TO \\
\hline \multirow[t]{2}{*}{ maturity } & high & low & low & high & high & high & high $^{\mathrm{b}}$ & low & high & high & low & low & high \\
\hline & \multicolumn{13}{|c|}{ Modelling Aspects } \\
\hline model type & $\mathrm{I}$ & I, D & I, D & I, D & I & $\mathrm{D}$ & $\mathrm{I}, \mathrm{D}^{\mathrm{b}}$ & I, D & $\mathrm{I}$ & $\mathrm{D}$ & $\mathrm{I}$ & I & $\mathrm{D}$ \\
\hline representation & $\begin{array}{l}\text { UML } \\
\text { XML }\end{array}$ & $\begin{array}{l}\text { UML } \\
\text { XML }\end{array}$ & $\begin{array}{l}\text { UML } \\
\text { XML }\end{array}$ & UML & UML & SMI & $\begin{array}{l}\text { RDF } \\
\text { XML } \\
\text { UML }\end{array}$ & $\begin{array}{l}\text { RDF } \\
\text { OWL }\end{array}$ & $\begin{array}{c}\text { NDL } \\
\text { RDF } \\
\text { VXDL } \\
\end{array}$ & \begin{tabular}{|c|} 
UML \\
XML \\
GRDF
\end{tabular} & XML & XML & $\begin{array}{l}\text { YANG } \\
\text { XML }\end{array}$ \\
\hline views & one & one & one & two & four & one & one & one & two & one & one & one & one \\
\hline policy & limited & limited & no & limited & yes & limited & no & yes & no & no & limited & no & yes \\
\hline context & no & no & no & no & yes & no & yes $^{\mathrm{c}}$ & yes & yes & no & no & no & no \\
\hline capability & yes & no & no & no & yes & no & no & yes & no & no & no & no & no \\
\hline \multirow[t]{2}{*}{ state } & limited & limited & no & no & yes & limited & no & yes & yes & no & no & no & yes \\
\hline & \multicolumn{13}{|c|}{ System Virtualization Perspective } \\
\hline $\begin{array}{l}\text { virtualization } \\
\text { concepts }\end{array}$ & yes & yes & yes & no & yes & yes & $\mathrm{yes}^{\mathrm{c}}$ & yes & yes & no & no & no & no \\
\hline hypervisor & indirect $t^{\mathrm{d}}$ & no & yes & no & indirect $^{\mathrm{d}}$ & indirect $^{\mathrm{d}}$ & no & no & no & no & no & no & no \\
\hline $\begin{array}{l}\text { hypervisor } \\
\text { agnostic }\end{array}$ & yes $^{d}$ & N/A & yes & N/A & yes $^{d}$ & yes $^{d}$ & $\mathrm{~N} / \mathrm{A}$ & N/A & N/A & N/A & N/A & $\mathrm{N} / \mathrm{A}$ & N/A \\
\hline
\end{tabular}

\subsection{Variety of modelled resources}

All proposals do abstract various resources, ranging from technical elements [38,44], to SLAs [96], relationships and roles [35,92], and business contexts [31,35]. Some proposals employ a broader scope abstracting a larger array of resources $[33,35,36]$, whereas others are more limited in that respect [97]. Assessment based on the modeled resources, thus, exhibits a higher degree of commonality.

A common drawback of all surveyed work is the lack of proper (explicit and detailed) hypervisor [52] abstraction. This is a serious drawback given recent trends in networking where the latter converges with computing. Most models treat hypervisors as transparent elements of the virtualization layer and begin abstracting form the virtual system or virtual network point. The sole exception is the KF model [38] that does contain hypervisor and hypervisor virtual switch classes but only includes introductory support and does not go into specific details. Partial and indirect support can be found in CIM, DEN-ng as well as in MIBs [44]. In CIM, a hypervisor (not a virtual machine) can be instantiated as a subclass via the OperatingSystem class and the built-in hypervisor virtual switch, respectively, via the UnitaryComputerSystem class. Although CIM (in the System Virtualization Model [55]) elaborates on modeling and management actions on a virtual machine and on the host computer system, it does not account for the hypervisor layer. DEN-ng, in a similar fashion, could be extended via subclassing from either the PhysicalResource and LogicalResource or the VirtualSystem and VirtualImage classes. In the MIBs domain the only relevant references are the VM-MIB [110] and the VMM-MIB [111], both at IETF draft status. These MIB objects can store basic hypervisor information (list of guest virtual machines, virtual CPU information and mappings of logical storage and network interfaces). Hypervisor technologies incorporate several operational specifics than what can be abstracted by available models $[112,114]$. Only recently has the need of modeling the hypervisor been, indirectly, acknowledged, in [113]. Three implementation examples, evolved around hypervisors, have resulted in CIM Schema extensions focusing on virtual machine monitoring and management. Microsoft's Hyper-V WMI Provider [125] exposes a CIM-based interface permitting users to monitor and control virtual machines hosted on a Hyper-V server. VMWare's 
CIM SMASH/Server Management API [115] is the equivalent product for the ESXi hypervisor and allows CIM-compliant management applications to manage the hypervisor and its virtual machines. Finally, Libvirt-CIM [116] is a CIM provider (originating from the Open Source community) for managing Linux virtualization platforms using the libvirt library. This product implements the virtualization class model from DMTF CIM's Experimental Schema and, mainly, focuses on managing the XEN [117] hypervisor and the virtual machines hosted by it.

\subsection{The need for actual implementations}

If there is a need for the target model to be applied in real world applications then it is clear that its instrumentation must be supported on the actual hardware and software environment where it will be applied. Therefore, choosing one of the proposed approaches based on possibility for instrumentation requires a mature model with collateral device support, also using well adopted and accepted representation methods. Here the choices are clearer: the broadest industry support is enjoyed by CIM, SID, SDN, DEN-ng and MIBs in the sense that there already exist management applications which employ the aforementioned models and can be expanded to suit custom infrastructures. Likewise, particular NDLs have been widely adopted and applied in various environments $[75,83]$. Elaborating on the maturity of each proposed model it is obvious that well established methods can increase the possibility for adoption. Restrictions still apply: CIM-based solutions are bound to the availability of specific operating system providers that materialize the instrumentation of a model element. If these providers are not available they will need to be developed, imposing an overhead in model adoption and solution provisioning. In a similar fashion, MIBs are implementation dependent and any solution relies on the availability of the suitable MIB modules, as discussed earlier. Finally, SDN is enjoying considerable industry support, making it a positive choice implementation-wise.

\subsection{Modelling perspective of surveyed proposals}

From a modeling perspective, the main models have been designed to cover a broad scope; hence incorporate greater detail and more modeling mechanisms than the rest of the proposals. SID partially originates from an older release of DEN-ng and this has resulted in the two models sharing, up to a certain extent, common concepts in model creation (relationships, attributes etc.) Both models differ from CIM in that different modeling approaches (meta-models etc.) are used. SID and DEN-ng are pure information models where-as CIM is not, as it is not entirely technology agnostic [50]. CIM provides for information abstraction that can be extended to include new items of the infrastructure and can be adapted to changes in management protocols. The model, however, does not include semantics for business processes and logic as do the other two main models. On the other hand, MIBs are technology-dependent data models that represent virtual containers for managed objects and their information. MIBs are much focused [118] and limited in scope, most often abstracting the specifics of a particular device or protocol. The three main models are object oriented in design where-as MIBs are hierarchical tree views of the managed objects. This implies that extending SID, DEN-ng and CIM presupposes a clear understanding of parts of the model, their class inheritance and associations. Extensions in MIBs are done by means of adding sub-trees to the hierarchical structure; a simpler process per se, partly accounting for MIBs and SNMP popularity.

Representation methods vary in the proposals - most use UML and XML (or clones of it), both mature and very widely accepted standards [53,74]. MIBs employ IETF's SMI standard (Structure of Management Information Version 2) [119], a subset of the ASN.1 standard (Abstract Syntax Notation One) [120]. SMI includes module, object and notification definitions for describing information semantics, managed objects' description and management information transmission respectively. SMI is inherently limited in semantic representation and cannot 
support complex data structures; hence, MIBs are bound by this restriction. This is hindered even more as MIBs use vendor specific data (by sub-tree addition for SNMP extensions) resulting in less standardization, even though SMI has been defined as a standard. On the other hand, SID and DEN-ng use UML (Unified Modeling Language). UML is far richer in semantic capabilities and techniques than SMI and can be used to construct and support complex models. CIM, although expressed and maintained in UML (and leveraging its advantages) does not result in fully compliant UML models. Information and concept expression in UML leverages the advantages of XML representation, thus the possibility of being used by a wider array of management (or other) applications. The NOVI [41], LICL [45] and SDN [46] models are exceptions to the general rule and use RDF/Owl, NDL/VXDL and YANG respectively.

SID and DEN-ng provide more modeling features, such as patterns and roles. Patterns provide for design reuse and roles provide abstractions for managed elements based on functions that they can perform. Several elements can be abstracted via roles, ranging from people to infrastructure item, such as devices. CIM does not support patterns or roles [121,123]. Another important quality characteristic between the three main models is their support for state (defined as the condition of any managed object at any given instance), context and policy. DEN-ng outweighs CIM [122] and SID in these features. Regarding state, most information models are referred to as current state models, i.e. they abstract and capture the state of a managed resource at a particular point in time and do not incorporate mechanisms for handling variations in state. CIM, via the CIMState extension together with specific properties and their enumerators, incorporates the current operational state of a managed resource. A common state model of CIM objects is provided via predefined states, where state pertains to managed object's attributes that can be queried or measured. DEN-ng includes advanced semantics and mechanisms $[59,123]$ and can provide for context and policy aware systems with varying object state depending on changes in context and policy. SID does not provide advanced support context or policy. A detailed discussion of these features and limitations can be found in $[60,61,63]$. DEN-ng, compared to the other two main information models (DMTF CIM and TMF SID), is superior in the sense that it is truly implementation independent and does include extensive features for policy, state and context representation. Additionally, DEN-ng provides a well-designed metadata model, whereas the other two models lack that feature. The minimal approach in class structure enables clearer design and better understanding of the model (in contrast to CIM and SID where thousands of classes are used). The NOVI information model [41] is, also, rich in features as it supports policy, context, capability and state. LICL [45], too, provides context-aware decisions and state capturing of infrastructure resources.

The rest of the surveyed approaches are limited from a modelling angle and this is to be expected as these models focus on a specific research aspect and have not been designed for a broader information abstraction scope. Information models are proposed in [96,97] for the management of SLA-based provisioning scenarios and virtual switches respectively. An information model, based on CIM, that abstracts system and network components found in a virtual network environment is proposed in [39]. Virtual machines and hypervisors are in the scope of work proposed in [38]. The semantics in these models allow for the description of different parts of the network virtualization environment; each model applies a different view on the modelled environment. None of these proposals, however, includes appropriate support for policy, context and capability; view support is limited too and in certain cases only. Finally, network description languages are mainly network-oriented [43]; therefore, they do not provide semantics and mechanism for describing computing architectures. This has been, recently, changed [91] with the proposal of INDL, which provides support for basic computing infrastructure. 
International Journal of Computer Networks \& Communications (IJCNC) Vol.6, No.3, May 2014

\section{Conclusions}

It is widely accepted in the networking community that network virtualization plays an important role in overcoming specific problems of the Internet and other large scale networks [2,3]. Moreover, relevant technological advancements introduced virtualized computing servers (in particular, their hypervisors being the network's "last-hop switch") as an integral part of computer networks [11], further complicating the network's architecture, operation and specifics. The standardization of the representation of network virtualization environments can be a solution to this ever increasing complexity and can, better, facilitate the management of such infrastructures. In this paper we surveyed information and data models available in the virtual network and system virtualization context. We grouped and structured known results and approaches so as to provide the reader with a generalized overview for comparing proposals on the topic (Table 1). Modern networks can be multidimensional entities with respect to the nature, role and function of their parts. Given the diverse nature of the involved architectures and resources no single approach can be best applied for abstracting this large collective of information. Ultimately, choosing a model for use in managing a, host-based virtual network infrastructure will depend on a number of factors, both modelling-related and other.

\section{REFERENCES}

[1] T.-R. Banniza et. al., (2009) "A European Approach to a Clean Slate Design for the Future Internet", Bell Labs Technical Journal, Vol. 14, No. 2, pp. 5-22.

[2] J. Carapinha, J. Jimenez, (2009) "Network Virtualization: a View from the Bottom", Proc. of the 1st ACM workshop on virtualized infrastructure systems/architectures, Barcelona, pp. 73-80.

[3] N. Niebert, I. Khayat, S. Baucke, R. Keller, R. Rembarz, and J. Sachs, (2008) "Network Virtualization: A viable path towards the future Internet", Wireless Personal Communications, Vol. 45, No. 4, pp. 511-520.

[4] T. Anderson, L. Peterson, S. Shenker, and J. Turner, (2005) "Overcoming the Internet impasse through network virtualization", Computer, Vol. 38, No. 4, pp. 34-41.

[5] N. M. M. K. Chowdhury and R. Boutaba, (2009) "Network Virtualization: State of the Art and Research Challenges", IEEE Communications Magazine, Vol. 47, No. 7, pp.20-26.

[6] N. M. M. K. Chowdhury, R. Boutaba, (2010) "A survey of network virtualization", Computer Networks, Vol. 54, No. 5, pp. 862-876.

[7] A. Botta, R. Canonico, G. Stasi, A. Pescape, G. Ventre, and S. Fdida, (2010) "Integration of 3G connectivity in PlanetLab Europe", Mobile. Networks Applications, Vol. 15, No. 3, pp. 344-355.

[8] F. Galan, D. Fernandez, W. Fuertes, M. Gomez, and J. Vergara, (2010) "Scenario-based virtual infrastructure management in research and educational testbeds with VNUML", Annals of Telecommunications, Vol. 64, No. 5-6, pp. 305-323.

[9] K. Tutschku, P. Tran-Gia, F. U. Andersen, (2008) "Trends in network and service operation for the emerging future Internet", International Journal of Electronic Communications, Vol. 62, No. 9, pp. 705-714.

[10] R. Canonico, D. Emma, and G. Ventre, (2008) "An XML Description Language for Web-Based Network Simulation", Proc. of the 7th IEEE International Symposium on Distributed Simulation and Real-Time Applications, Delft, The Netherlands, pp. 76-81.

[11] M. Creeger, (2010) "Moving to the edge: a CTO roundtable on network virtualization", Communications of the ACM, Vol. 53, No. 8, pp. 55-62.

[12] IBM, (2013) "Virtual I/O Server", https://www-304.ibm.com/webapp/set2/sas/f/vios/home.html

[13] IBM, (2013) "Server virtualization with IBM PowerVM", http://www03.ibm.com/systems/power/software/virtualization

[14] M. Armbrust, et. al., (2009) "Above the Clouds: A Berkeley View of Cloud Computing", University of California, Berkeley, Dept. Electrical Eng. and Comput. Sciences, Report UCB/EECS 28:13.

[15] C. E. Perkins, (2002) "Mobile IP", IEEE Communications Magazine, Vol. 35, No. 5, pp. 84-89.

[16] F. Baroncelli, B. Martini, and P. Castoldi, (2010) "Network virtualization for cloud computing", Annals of Telecommunications, Vol. 65, No. 11-12, pp. 713-721. 
[17] W. Haerick, T. Wauters, C. Develder, F. Turck, and B. Dhoedt, (2010) "Transparent resource sharing framework for internet services on handheld devices", Annals of Telecommunications, Vol. 65, No. 7-8, pp. 419-432.

[18] Kumar, S., (2014) "Ubiquitous Smart Home System Using Android Application", International Journal of Computer Networks \& Communications, Vol.6, No.1, pp. 33-43

[19] K. Kroeker, (2009) "The evolution of virtualization", Communications of the ACM, Vol. 52, No. 3, pp. 18-20.

[20] E. Herscovitz, (1999) "Secure Virtual Private Networks: The Future of Data Communications", International Journal of Network Management, Vol. 9, No. 4, pp. 213-220.

[21] Sleit, A. et al., (2013) "Cloud Computing Challenges With Emphasis On Amazon EC2 and Windows AZURE", International Journal of Computer Networks \& Communications, Vol. 5, No.5, pp. 35-44.

[22] Shin-ichi Kuribayashi, (2013) "Joint Multiple Resource Allocation Method for Cloud Computing Services with Different QOS to Users at Multiple Locations", International Journal of Computer Networks \& Communications, Vol. 5, No.5, pp. 01-18.

[23] D. L. Tennenhouse and D. J. Wetherall, (2007) "Towards an active network architecture", ACM SIGCOMM Computer Communication Review, Vol. 37, No. 5, pp. 81-94.

[24] A. T. Campbell et. al., (1999) "A Survey of Programmable Networks", ACM SIGCOMM Computer Communication Review, Vol. 29, No. 2, pp. 7-23.

[25] D. Andersen, H. Balakrishnan, F. Kaashoek, and R. Morris, (2001) "Resilient Overlay Networks", ACM SIGCOMM Computer Communication Review, Vol. 32, No. 1, pp 66.

[26] J. Touch and S. Hotz, (1998) "The X-Bone", Proc. of the IEEE 3rd Global Internet Mini-Conference in conjunction with GLOBECOMM98, Sydney.

[27] N. M. M. K. Chowdhury, F. Zaheer, and R. Boutaba, (2009) "iMark: An Identity Management Framework for Network Virtualization Environment", Proc. of the IFIP/IEEE International Symposium on Integrated Network Management, New York, pp. 335-342.

[28] G. Schaffrath et. al., (2009) "Network Virtualization Architecture: Proposal and Initial Prototype", Proc. of the 1st ACM workshop on Virtualized Infrastructure Systems and Architectures, Barcelona, pp. 63-72.

[29] M. Boucadair, P. Georgatsos, N. Wang, D. Griffin, G. Pavlou, M. Howarth, and A. Elizondo, (2009) "The AGAVE approach for network virtualization: differentiated services delivery", Annals of Telecommunications, Vol. 64, No. 5-6, pp. 277-288.

[30] N. Feamster, L. Gao, and J. Rexford, (2007) "How to lease the Internet in your spare time", ACM SIGCOMM Computer Communication Review, Vol. 37, No. 1, pp. 61-64.

[31] K. Oberle, M. Kessler, M. Stein, T. Voith, D. Lamp, and S. Berger, (2009) "Network virtualization: the missing piece", Proc. of the IEEE 13th International Conference on Intelligence in Next Generation Networks, Bordeaux, pp. 1-6.

[32] European Union IRMOS project, (2011) "Interactive Real-time Multimedia Applications on Service Oriented Infrastructures", http://www.irmosproject.eu/

[33] Distributed Management Task Force, (2011) "Common Information Model CIM", DMTF Specification DSP0004, http://www.dmtf.org/standards/cim

[34] Distributed Management Task Force, (2013) http://www.dmtf.org/

[35] TMForum, (2013) "The Information Framework (SID)", http://tmforum.org/InformationFramework/1684/home.html

[36] J. Strassner, (2002) "DEN-ng: achieving business-driven network management", Proc. of IEEE/IFIP Network Operations and Management Symposium, Colorado Springs, pp. 753-766.

[37] The Autonomic Communications Forum, (2012) http://www.autonomic-communication.org/

[38] D. Kontoudis and P. Fouliras, (2013) "Modelling and managing virtual network environments", Proc. of the 17th PanHellenic Conference on Informatics, Thessaloniki, pp. 39-46.

[39] W. Fuertes, J. E. V. Lopez, F. Meneses, and F. Galan, (2010) "A generic model for the management of virtual network environments". Proc. of the IEEE Network Operations and Management Symposium, Osaka, pp. 813-816.

[40] N. Agoulmine, (2010) Autonomic Network Management Principles, Academic Press, ISBN 978-012-382190-4.

[41] P. Grosso et. al, (2011) "Information models for virtualized architectures - NOVI", Deliverable D2.111.02.28-v3.1, http://www.fp7-novi.eu/deliverables/doc_download/25-d21

[42] J. Van der Ham et. al, (2011) "Challenges of an information model for federating virtualized infrastructures", Proc. of the 5th International DMTF Academic Alliance Workshop on Systems and Virtualization Management: Standards and the Cloud, Paris, pp. 1-6. 
International Journal of Computer Networks \& Communications (IJCNC) Vol.6, No.3, May 2014

[43] ERCIM, (2009) "Network description tools and standards", ERCIM News, Vol. 77, No. 1, pp. 33-34.

[44] B. Fenner, (2012) "MIB index", http://www.icir.org/fenner/mibs/mib-index.html

[45] J. A. Garcia-Espin et. al., (2010) "Functional Description of the Logical Infrastructure Composition Layer ", http://www.geysers. eu/images/stories/deliverables/geysers-deliverable_3.1.pdf

[47] Open Networking Foundation, (2012) "Software-Defined Networking: The New Norm for Networks", $\quad$ https://www.opennetworking.org/images/stories/downloads/openflow/wp-sdnnewnorm.pdf

[47] K. Hyojoon and N. Feamster, (2013) "Improving network management with software defined networking", IEEE Communications Magazine, Vol. 51, No. 2, pp. 114-119.

[48] A. Devlic, W. John, and P. Skoldstrom, (2012) "A Use-Case Based Analysis of Network Management Functions in the ONF SDN Model", Proc. of the 2012 European Workshop on Software Defined Networking (EWSDN), Darmstadt, pp. 85-90.

[49] J. Mylopoulos, (1998) "Information Modelling in the Time of the Revolution", Information Systems, Vol. 23, No. 3-4, pp. 127-155.

[50] J. Strassner, (2003) Policy-based Network Management: Solutions for the Next Generation, Morgan Kaufman, ISBN 978-1558608597.

[51] J. Jiyong, H. Saeyoung, K. Jinseok, P. Sungyong, B. Seungjo, and C. W. Young, (2007) "A Performance Evaluation Methodology in Virtual Environments", Proc. of the 7th IEEE International Conference on Computer and Information Technology, Fukushima, pp. 351-358.

[52] W. Armstrong, R. Arndt, D. Boutcher, R. Kovacs, D. Larson, K. Lucke, N. Nayar, and R. Swanberg, (2005) "Advanced virtualization capabilities of POWER5 systems", IBM Journal of Research and Development, Vol. 49, No. 4, pp. 523-532.

[53] Open Management Group, (2013) "Unified Modeling Language (UML)", http://www.uml.org/

[54] Distributed Management Task Force, (2003) "CIM Network Model", White Paper DSP0152, http://www.dmtf.org/sites/default/files/standards/documents/DSP0152.pdf

[55] Distributed Management Task Force, (2007) "CIM System Virtualization Profile", Informational Standard DSP2013, http://www.dmtf.org/standards/published_documents/DSP2013_1.0.0.pdf

[56] Distributed Management Task Force, (2003) "CIM Metrics Model", White Paper DSP0141, http://www.dmtf.org/sites/default/files/standards/documents/DSP0141.pdf

[57] The Telecommunication Management Forum, (2013) http://www.tmforum.org

[58] The Telecommunication Management Forum, (2012) "TMForum Frameworx", http://www.tmforum.org/TMForumFrameworx/1911/home.html

[59] J. Strassner, (2006) "FOCALE - A Novel Autonomic Computing Architecture", Proc. of the Latin American Autonomic Computing Symposium (LAACS), Campo Grande.

[60] D. Raymer, J. Strassner, E. Lehtihet, and S. Van der Meer, (2006) "End-to-End Model Driven Policy Based Network Management", Proc. of the 7th IEEE International Workshop on Policies for Distributed Systems and Networks, Ontario, pp. 4-70.

[61] J. Strassner, S. Van der Meer, D. O 'Sullivan, and S. Dobson, (2009) "The Use of Context-Aware Policies and Ontologies to Facilitate Business-Aware Network Management", Journal of Network and Systems Management, Vol. 17, No. 3, pp. 225-284.

[62] B. Moore, E. Ellesson, J. Strassner, and A. Westerinen, (2001) "Policy core information model", IETF Network Working Group RFC RFC3060, http://www.ietf.org/rfc/rfc3060.txt

[63] J. Strassner et. al, (2008) "Modelling Context for Autonomic Networking", Proc. of the IEEE Network Operations and Management Symposium, Salvador da Bahia, pp. 299-308.

[64] European Union 7th FP, (2012) "The AUTOI project", http://ist-autoi.eu

[65] C. Fahy et. al., (2008) "Towards an information model that supports service-aware, self-managing virtual resources", Proc. of the Modeling autonomic communications environments conference (MACE), pp. 102-107.

[66] IEEE 802.1 SMIv2 MIB Modules, (2013), http://www.ieee802.org/1/pages/MIBS.html

[67] IETF MIBs, (2013), http://www.simpleweb.org/ietf/mibs/index.php?sel=IETF

[68] A. Bierman and K. Jones, (2000) "Physical Topology MIB", RFC 2922, http://gamay.tools.ietf.org/html/rfc2922

[69] K. McCloghrie and A. Bierman, (1999) "Entity MIB (Version 2)", IETF RFC2737, http://tools.ietf.org/html/rfc2737

[70] C. Srinivasan, L. P. Bloomberg, A. Viswanathan, and T. Nadeau, (2004) "Multiprotocol Label Switching Traffic Engineering Management Information Base", IETF RFC3812, http://www.ietf.org/rfc/rfc3812.txt 
International Journal of Computer Networks \& Communications (IJCNC) Vol.6, No.3, May 2014

[71] W. Ng, D. Jun, R. Chow, R. Boutaba, and A. Leon-Garcia, (1999) "MIBlets: a practical approach to virtual network management", Proc. of the 6th IFIP/IEEE International Symposium on Integrated Network Management, Boston, pp. 201-215.

[72] E. Stelzer, S. Hancock, B. Schliesser, and J. Laria, (2005) "Virtual Router Management Information Base Using SMIv2", IETF Draft, http://tools.ietf.org/html/draft-ietf-13vpn-vr-mib-04

[73] K. McCloghrie and A. Bierman, (1996) "Entity MIB using SMIv2", IETF RFC2037, http://tools.ietf.org/html/rfc2037

[74] T. Bray, J. Paoli, C. M. Sperberg-McQueen, F. Yergeau, and J. Cowan, (2006) "Extensible Markup Language (XML)", http://www.w3pdf.com/W3cSpec/XML/2/REC-xml11-20060816.pdf

[75] University of Amsterdam SNE research group, (2013) "Network Description Language", http://www.science.uva.nl/research/sne/ndl

[76] World Wide Web Consortium W3C, (2013), http://www.w3c.org

[77] Global Lamda Integrated Facility, (2013), http://www.glif.is

[78] University of Amsterdam SNE research group, (2013) "Device description using NDL, Multi-layer NDL configuration files", http://ndl.uva.netherlight.nl/config.html

[79] J. Van der Ham, (2013) "Map creation with NDL", http://staff.science.uva.nl/ vdham/NDL/googlemap.html

[80] F. Dijkstra, (2007) "Multi-Layer Path Finding with NDL", http://ndl.uva.netherlight.nl/Glif-DemoSep07

[81] NML Working Group, (2013), "Network Markup Language", http://www.ogf.org/gf/group_info/areasgroups.php?area_id=1

[82] Open Grid Forum, (2010), http://www.ogf.org

[83] NML Working Group Home, (2010), https://forge.gridforum.org/sf/projects/nml-wg

[84] J. Van der Ham, F. Dijkstra, R. Lapacz, and J. Zurawski, (2012) "Network Markup Language Base Schema, wg/docman.root.drafts/doc 15674

[85] Rice University, (2009) "The Virtual Grid Description Language: vgDL", http://vgrads.rice.edu/publications/vgdltr

[86] L. Ramakrishnan et. al., (2009) "VGrADS: Enabling e-Science Workflows on Grids and Clouds with Fault Tolerance", Proc. of the SC09 High Performance Computing Networking, Storage and Analysis Conference, New York, pp. 1-12.

[87] Rice University, (2009) "vgDL Applications", http://vgrads.rice.edu/research/applications

[88] G. P. Koslovski, P. V. B. Primet, A. S. Charao, (2009) "VXDL: Virtual Resources and Interconnection Description Language", Networks Grid Applications, Vol. 2, No. 1, pp. 138-154.

[89] G. Koslovski, T. T. Huu, J. Montagnat, and P. Vicat-Blanc, (2010) "Executing distributed applications on virtualized infrastructures specified with the VXDL language and managed by the HIPerNET framework", Lecture Notes of the Institute for Computer Sciences, Social-Informatics and Telecommunications Engineering, Vol. 1, No. 1, pp. 3-19.

[90] F. Anhalt, G. Koslovski, and P. V. B. Primet, (2010) "Specifying and provisioning virtual infrastructures with HIPerNET", International Journal of Network Management, Vol. 20, No. 3, pp. 129-148.

[91] M. Ghijsen, J. Van der Ham, P. Grosso, and C. De Laat, (2012) "Towards an Infrastructure Description Language for Modeling Computing Infrastructures", Proc. of the 10th IEEE International Symposium on Parallel and Distributed Processing with Applications, Madrid, pp. 207-214.

[92] L. Volker, D. Martin, I. Khayaty, C. Werle, and M. Zitterbart, (2009) "A Node Architecture for 1000 Future Networks", Proc. of the Intenational Conference on Communications Workshops, pp. 1-5.

[93] European Union FP7 4WARD Project, (2011), http://www.4ward-project.eu

[94] B. Ahlgren, C. Dannewitz, C. Imbrenda, D. Kutscher, and B. Ohlman, (2012) "A survey of information-centric networking", IEEE Communications Magazine, Vol. 50, No. 7, pp. 26-36.

[95] European Union SAIL Project, (2012), http://www.sail-project.eu

[96] I. Fajjari, M. Ayari, and G. Pujolle, (2010) "VN-SLA: A Virtual Network Specification Schema for Virtual Network Provisioning", Proc. of the IEEE 9th International Conference on Networks, Menuires, pp. 337-342.

[97] H. Okita, M. Yoshizawa, T. Suzuki, and T. Iijima, (2011) "Virtual Network Management Information Model (VNMI)", IETF Draft, http://tools.ietf.org/html/draft-okita-ops-vnetmodel-05

[98] R. Wang, D. Butnariou, and J. Rexford, (2011) "OpenFlow-based server load balancing gone wild", Proc. of the Workshop on Hot Topics in Management of Internet, Cloud, and Enterprise Networks and Services (Hot-ICE), Boston, pp. 12-12. 
[99] M. Casado, T. Koponen, R. Ramathan, and S. Shenker, (2010) "Virtualizing the Network Forwarding Plane", Proc. of the Workshop on Programmable Routers for Extensible Services of Tomorrow, Philadelphia, USA.

[100]T. Koponen et. al., (2010) "Onix: A Distributed Control Platform for Large-scale Production Networks", Proc. of the 9th Usenix Conference on Operating Systems Design and Implementation, Vancouver, pp. 1-6.

[101]C. Rotsos, R. Mortier, A. Madhavapeddy, B. Singh, and A. Moore, (2012) "Cost, performance \& flexibility in OpenFlow: Pick three", Proc. of the SDN 2012 Workshop on Software Defined Networks, Ottawa, pp. 6601-6605.

[102]N. McKeown et. al., (2008) "OpenFlow: Enabling Innovation in Campus Networks", ACM SIGCOMM Computer Communication Review, Vol. 38, No. 2, pp. 67-94.

[103]R. Enns, (2006) "NETCONF Configuration Protocol", IETF RFC4741, http://www.ietf.org/rfc/rfc4741.txt

[104]M. Bjorklund, (2010) "YANG - A Data Modeling Language for the Network Configuration Protocol (NETCONF)", IETF RFC RFC6020, http://tools.ietf.org/html/rfc60202010

[105]Cisco Systems, (2011) "What is OverDrive Network Hypervisor?", http://www.cisco.com/en/US/docs/net_mgmt/datacenter_mgmt/OverDrive/4.0/Introducing/intro_over view.html

[106]Extreme Networks, (2013) "SDN: Software Defined Networking", http://www.extremenetworks.com/solutions/datacenter_sdn.aspx

[107]G. M. Giaglis, (2001) "A taxonomy of business process modelling and information systems modelling techniques", International Journal of Flexible Manufacturing, Vol. 13, No. 2, pp. 209-228.

[108]A. Gemino and Y. Wand, (2004) "A framework for empirical evaluation of conceptual modelling techniques", Requirements Engineering, Vol. 9, No. 4, pp. 248-260.

[109]European Union INTERMON Project, (2012), http://www.ist-intermon.org

[110]M. McFaden, J. Schoenwaelder, T. Tsou, and C. Zhou, (2012) "Definition of Managed Objects for Virtual Machines Controlled by a Hypervisor (Hypervisor MIB)", IETF Draft, http://tools.ietf.org/html/draft-schoenw-opsawg-vm-mib-01

[111]H. Asai, Y. Sekiya, K. Shima, and H. Esaki, (2013) "Management Information Base for the Virtual Machine Manager", IETF Draft, http://tools.ietf.org/html/draft-asai-vmm-mib-00

[112]M. Fenn, M. Murphy, J. Martin, and S. Goasguen, (2008) "An evaluation of KVM for use in cloud computing", Proc. of the 2nd International Conference on the Virtual Computing Initiative.

[113]V. A. Danciou, (2011) "Bottom-up harmonisation of management attributes describing hypervisors and virtual machines", Proc. of the 5th International DMTF Alliance Workshop, Paris, pp. 1-10.

[114]VMware, (2013) "vSphere Hypervisor (ESXi)", http://www.vmware.com/products/vspherehypervisor

[115]VMware, (2012) "CIM SMASH/Server Management API for ESXi", http://pubs.vmware.com/vsphere-50/index.jsp

[116]LibVirt CIM provider, (2013), http://libvirt.org/CIM/intro.html

[117]The Linux Foundation, (2013) "XEN Hypervisor", http://www.xenproject.org

[118]Network management RFCs sorted by SMI/MIB, (2013), http://www.simpleweb.org/ietf/rfcs/rfcbymodule.php

[119]K. McCloghrie, D. Perkins, and J. Schoenwaelder, (2013) "Structure of Management Information Version 2 (SMIv2)", IETF RFC 2578, http://tools.ietf.org/html/rfc2578

[120]ITU, (2002) "Information technology - Abstract Syntax Notation One (ASN.1)", ITU-T Recommendation X.680, http://www.itu.int/ITU-T/studygroups/com17/languages/X.680-0207.pdf

[121]Y. Choi, J. Li, Y. Han, J. Strassner, and J. W. K. Hong, (2010) "Towards a Context-Aware Information Model for Provisioning and Managing Virtual Resources and Services", Proc. of the Modeling Autonomic Communication Environments conference (MACE '10), pp. 100-112.

[122] Distributed Management Task Force, (2003) "CIM Policy Model", DMTF White Paper DSP0108,http://dmtf.org/documents/cim-policy-white-paper-270

[123] J. Strassner, S. Van der Meer, and J. W. K. Hong, (2009) "The Applicability of Self-Awareness for Network Management Operations", Proc. of the Modelling Autonomic Communication Environments conference (MACE '09), pp. 15-28.

[124]European Union GEYSERS Project, (2013), http://www.geysers.eu

[125] Microsoft, (2013) "Hyper-V WMI Provider", http://msdn.microsoft.com/enus/library/hh850013\%28v=vs.85\%29.aspx 
International Journal of Computer Networks \& Communications (IJCNC) Vol.6, No.3, May 2014

\section{Authors}

Dimitris Kontoudis is a Ph.D. candidate at the University of Macedonia, Greece, and a deputy director with the Information Technology division of the National Bank of Greece. He holds a B.Sc. in Physics and Scientific Computing (University of Greenwich, UK) and an M.Sc. in Advanced Methods in Computer Science (University of London, UK). His research interests include systems/network virtualization and data centre infrastructure performance management.

Dr. Panayotis Fouliras is Assistant Professor at the Department of Applied Informatics at the University of Macedonia, Thessaloniki, Greece. He obtained his B.Sc. in Physics (Aristotle University of Thessaloniki, Greece), M.Sc. and Ph.D in Computer Science from University of London, UK (QMW). His research interests span computer networks, QoS, multimedia and system evaluation methods.

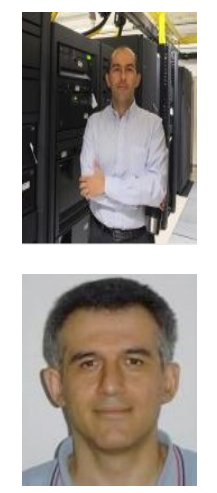

